\title{
Demand contracting strategy for charging stations based on load forecasting
}

\author{
Fang Chen ${ }^{1}$, Liu Zeyu ${ }^{2}$, Wang Haojing ${ }^{1}$, Zhao $\mathrm{Yi}^{2}$, Shi Shanshan ${ }^{1}$ and Gui Qiang ${ }^{2}$ \\ ${ }^{1}$ States Grid Shanghai Municipal Electric Power Company, 200120, Shanghai, China \\ ${ }^{2}$ Shanghai Jiao Tong University, 200240, Shanghai, China
}

\begin{abstract}
Since the stochasticity of the charging of electric vehicles (EVs) may bring impact to the grid, there is a high possibility that the demand charge will be applied to charging stations. Therefore, a loadforecasting-based demand contracting strategy is proposed for charge stations in this paper. A stochastic optimization model is established by regarding the maximal demand as a stochastic parameter, and the object of the model is to minimize the expectation of demand charge, and the analytic solution is derived. To obtain the distribution of actual maximal demand, a Monte-Carlo-based charge load forecasting method is proposed. It gives the distribution of the daily maximal demand, based on which the distribution of monthly maximal demand is also derived. The case study illustrates the feasibility and the validity of the proposed strategy.
\end{abstract}

\section{Introduction}

In recent years, countries around the world have paid attention to environmental pollution including air pollution, one of the important sources of which is the traditional autos using internal combustion engines. Since electric vehicles (EVs) are driven by electric energy, no fossil fuels are burned directly to emit exhaust gas. Besides, EVs have the advantages of low noise, so they are regarded as "clean vehicles" and are ideal alternatives to internal combustion engine vehicles.

The number of electric vehicles has been increasing in recent years, which puts forward higher requirements for the development of electric vehicle charging facilities. Nowadays, "mileage anxiety" is still one of the main reasons that restrict people from buying electric vehicles, so convenient and fast charging has become an important thrust to promote the development of the electric vehicle industry. For this reason, many countries are vigorously developing the electric vehicle industry and infrastructure construction. The Ministry of Industry and Information Technology of the People's Republic of China proposed that by 2025 , the sales ratio of new energy vehicles will reach about $25 \%$, and it clearly states that it must "improve infrastructure construction" and "improve the level of charging infrastructure services." ${ }^{[1]}$. The electric vehicle industry has the same phenomenon abroad ${ }^{[2-3]}$. In 2016, Norway built the world's largest electric vehicle fast charging station. London has built more than 225 fast charging stations in 2019. EVgo, which is the electric vehicle charging network operator in American is advancing the construction of fast charging stations with battery energy storage systems. In terms of electricity prices, many countries in the world have implemented a segmented charging model ${ }^{[4-5]}$. In 2014, the National Development and Reform Commission of China made it clear that large-scale industrial electricity prices would be applied to the centralized charging facilities, and the basic electricity charges would be waived before $2020^{[6]}$.

The basic electricity charge is a form of electricity fee charged for large users with a transformer capacity of $100 \mathrm{kVA}$ and above. This kind of users need to implement a two-part electricity price, including electricity price (charged based on electricity consumption) and basic electricity price (charged based on maximum power) ${ }^{[7]}$. The basic electricity charge can be charged in two ways: according to the transformer capacity or the maximal demand, which can be freely selected by users. It is generally believed that charging the basic electricity fee based on the maximal demand (referred to as the demand charge) is a more reasonable method $^{[8]}$. The main purpose of the basic electricity charge is to promote the rational use of electricity and limit the short-term load spikes generated by high-power users $^{[9]}$. The charging of electric vehicles is stochastic, and there are quite a few studies to point out that largescale electric vehicle charging may bring impact to the grid $^{[10-15]}$. Measures need to be taken to limit the shortterm high-power operation of charging stations, which is in line with the concept of demand charge. Although charging stations are tentatively exempted from the basic electricity charge in order to promote the development of electric vehicle charging infrastructure, in the foreseeable future, they are very likely to become one of the charging objects of the basic electricity bill in order to promote the safety and stability of the grid.

\footnotetext{
* Corresponding author: liuzeyu@sjtu.edu.cn
} 
In this context, electricity expenses will be the one of the major costs of charging stations, of which demand charge will be a significant part. When demand charge is charged, the operator should sign a contract with the grid company to declare its own "contract demand" (this process can be referred to as "demand contracting"), and be charged according to the contract demand. However, if the actual maximal demand exceeds 5\% above the contract demand, the excessive part will be charged by doubled price. Therefore, a reasonable choice of the contract demand will have a significant effect on the cost reduction of the charging station.

Based on the above background, this paper studies the demand contracting strategy for charging stations and proposes a load-forecasting-based demand contracting strategy. This paper establishes a mathematical model of the demand charge based on the current demand charge rules, and derives the optimal solution for the contract demand through mathematical analysis. Since the effectiveness of the above demand contracting strategy largely depends on whether the charging station operator can better obtain the distribution of the actual maximal demand, this paper proposes a load-forecasting-based demand contracting strategy. Furthermore, the method of obtaining the distribution of monthly maximal demand is given. Finally, the effectiveness of the method proposed in this paper is demonstrated through the analysis of a numerical study.

\section{Demand contracting strategy for charging stations}

According to the current regulations on the demand charge, when users calculate the basic electricity charge based on the maximal demand, a general formula of the basic electricity charge can be written as:

$$
\tilde{c}= \begin{cases}p_{1} x+p_{2}(\tilde{D}-k x), & k x<\tilde{D} \leq C \\ p_{1} x, & \tilde{D} \leq k x\end{cases}
$$

where $\tilde{c}$ presents the demand charge; $C$ is the capacity of the transformer; $\tilde{D}$ is the actual maximal demand of users; $x$ is the contract demand, obeying $\underline{D} \leq x \leq C ; \underline{D}$ is the minimal contract demand limit, obeying $\underline{D}=0.4 \mathrm{C} ; k$ is the threshold for charging a punitive price, which is $1.05 ; p_{1}, p_{2}$ are the price for the actual maximal demand within $k$ times of the contract demand (called the basic demand price) and the demand price for the part that exceeds $k$ times of the contract demand (called the punitive demand price) respectively, obeying $p_{2} / p_{1}=2$; however, the values of $p_{1}, p_{2}$ vary in different provinces.

Equation (1) shows that the demand charge is related to $C, \underline{D}, \tilde{D}, x, k, p_{1}, p_{2}$. For charging station operators, the only variable that is fully controllable is the contract demand $x$. Therefore, from the perspective of the contract demand, Equation (1) can be rewritten as:

$$
\tilde{c}= \begin{cases}\left(p_{1}-k p_{2}\right) x+p_{2} \tilde{D}, & \underline{D}<x<\tilde{D} / k \\ p_{1} x \quad, & x \geq \tilde{D} / k\end{cases}
$$

It can be seen that the demand contracting strategy for charging stations aims to determine the contract demand $x$ that can make the basic electricity charge minimized. However, the actual maximal demand of the charging station is a random parameter, which mainly depends on the charging status of the electric vehicle. Therefore, a stochastic optimization model is established by regarding the maximal demand as a stochastic parameter, the object of which is to minimize the expectation of demand charge.

$$
\begin{gathered}
\operatorname{Min}_{x} E[\tilde{c}(x)]=p_{1} x+\int_{k \cdot x}^{C} p_{2}(\tau-k x) f_{\tilde{D}}(\tau) \mathrm{d} \tau \\
\text { s.t. } \quad \underline{D} \leq x \leq C
\end{gathered}
$$

where $f_{\tilde{D}}(\bullet)$ presents the probability density function of the actual maximal demand $\tilde{D}$.

Through derivative analysis of the optimization problem expressed by equation (3) and equation (4), the analytical solution of the problem can be obtained as:

$$
x^{*}= \begin{cases}\underline{D}, & F_{\tilde{D}}(k \underline{D}) \geq \frac{p_{2} k-p_{1}}{p_{2} k} \\ \frac{F_{\tilde{D}}^{-1}\left[1-p_{1} /\left(p_{2} k\right)\right]}{k}, & F_{\tilde{D}}(k \underline{D})<\frac{p_{2} k-p_{1}}{p_{2} k}\end{cases}
$$

where $F_{\tilde{D}}(\bullet)$ presents the cumulative distribution function of the actual maximal demand $\tilde{D}$.

The specific derivation process can be found in literature [16]. When the probability that actual maximal demand of users is below $0.42 C$ is not less than 0.524 , the optimal value of the contract demand is equal to the minimal contracting limit $\underline{D}=0.4 C$; Otherwise, the optimal value of the contract demand is equal to $F_{\tilde{D}}^{-1}(0.524) / 1.05$.

\section{Demand contracting strategy for charging stations}

\subsection{NHTS data processing}

From the demand contracting strategy for charging stations obtained in Section 1, it can be known that whether users can better determine the contract demand depends largely on whether the user can predict a better distribution of the actual maximal demand. For electric vehicle charging stations, the electricity load mainly comes from the load of electric vehicles that charging at the charging station. Therefore, in order to obtain the maximal demand of the charging station in the future, the electric vehicle charging load forecast of the charging station is extremely important. In this section, we propose a Monte Carlo simulation method based on the trip chain theory to predict the charging load of the charging station.

The users of public charging stations are mainly household EVs and passenger EVs. For these vehicles, their service scenarios are usually people's daily travel. 
The behavior characteristics of this type of vehicles can be considered to have similarities with that of private cars. Therefore, the use of travel survey data for household petrol vehicles has an important value for this research. On account of the above considerations, the household travel survey data NHTS 2017 led by the US Federal Highway Administration is used as the data basis.

For the travel characteristics of the private car, various destinations can usually be divided into 5 categories according to the purpose of travel: $\mathrm{H}$ (home), W (work), SE (shopping \& errands), SR (social \& recreation), $\mathrm{O}$ (other). The closed loop among the five types of destinations and centered on $\mathrm{H}$ is called a trip chain. Since trip chains with too many midway destinations are minority, and usually include temporary parking (where charging behavior is usually unlikely to occur). Therefore, only trip chains with no more than 2 midway locations are considered. The classification of trip chains is shown in Fig. 1.

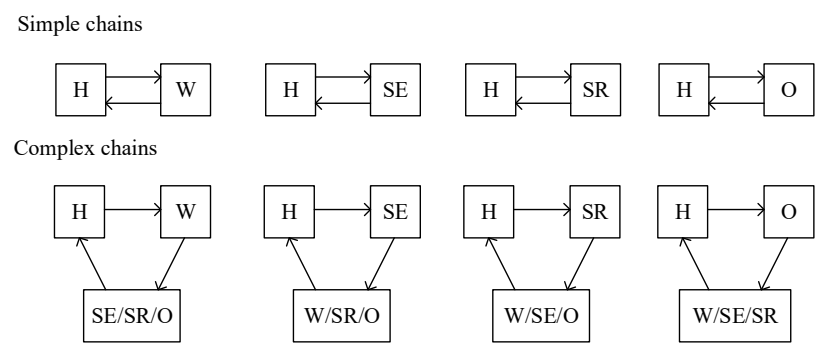

Fig. 1 Classification of trip chains

In NHTS 2017 data, about 923500 records of household trips with necessary information of each trip are recorded. The following steps are taken to analyse the characteristics of household EVs trips.

Step 1: Read the data of the current travel records.

Step 2: When all the necessary data for the closed trip chains is recorded, add it to the data set of the corresponding type of the trip chain.

Step 3: Accumulate the data of each trip of various trip chains in the data set, and use the kernel density estimation method to fit the probability density function.

Through the above data processing process, the following probability statistical results related to the electric vehicle trip chain can be obtained:

(1) The Distribution of the starting/ending time, the trip duration, the trip length, and average speed in each type of trip chains;

(2) The distribution of staying duration at every midway site of every type of trip chains.

(3) The proportion of each trip chain.

\subsection{Load forecasting method based on Monte Carlo simulation}

Based on the data processing of NHTS 2017, a statistical feature of trip characteristics is obtained. The whole trip chains of EVs are simulated in a way that is designed to be close to the real using scenarios. In the simulation, necessary parameters are generated according to the fitted distribution.
Specifically, the simulation process can be described as the following process:

Step 1: According to the proportion of various types of trip chains, randomly select the type of trip chains (travel purpose) for this EV trip.

Step 2: According to the corresponding type of the travel chain, randomly select the end time of the first leg of the trip. Considering that the start time of a day's schedule is relatively fixed (such as work time, the time appointed by customers or friends), the first moment to be generated is set to be the ending time of trip 1 .

Step 3: According to the random distribution of the distance and average speed in the current trip chain, select the distance and average speed of trip 1. The length of each trip is determined by the distance between the starting site and the destination, and is the main factor influencing power consumption. The average velocity, which is affected by factors including weather and traffic congestion, is the determinant for trip duration when length is fixed.

Step 4: According to the current type of the trip chain, select the staying duration at the midway site. It is generally believed that the charging behavior of EVs mostly occurs at the midway site. The principle of charging is to ensure the usage of EVs. Therefore, the EVs are set to be charged when the state of charge (SOC) is inadequate for the next trip. Considering a security redundancy of $20 \%$ SOC remained after the next trip, the $\mathrm{EV}$ is charged when:

$$
\mathrm{SOC}_{n}-u l_{n} / c \leq 0.2
$$

where $S O C_{n}$ is the SOC when reaching the $n$th site of the trip chain; $u$ is the power consumption per kilometer; $l_{n}$ is the length of the $n$th trip of the trip chain; $c$ is the battery capacity.

The EV's SOC at the $n$th site can be calculated by

$$
S O C_{n}=S O C_{n-1}-u l_{n-1}
$$

where the initial SOC $\left(S O C_{0}\right)$ is set to be 1 if the user owns a private charging post, or a random value in $[0.5,1]$ if the user does not. When an EV is charged at a site, the charging power of it is added to the charge load of this site during the whole charging duration. The charging duration at site $n$ is the minimum between staying time and the necessary charging time till the EV is fully charged.

$$
T_{\text {charge }, n}=\min \left\{T_{\text {stay }},\left(1-S O C_{n}\right) \cdot c / p\right\}
$$

where $p$ presents the charging power.

Step 5: If the EV's trip has not ended, repeat steps 3 and 4 until the EV's trip is completely simulated. Finally, the charging power is added on the charging load curve at point $H$.

The simulation procedure can be expressed as the flow chart in Fig. 2. 


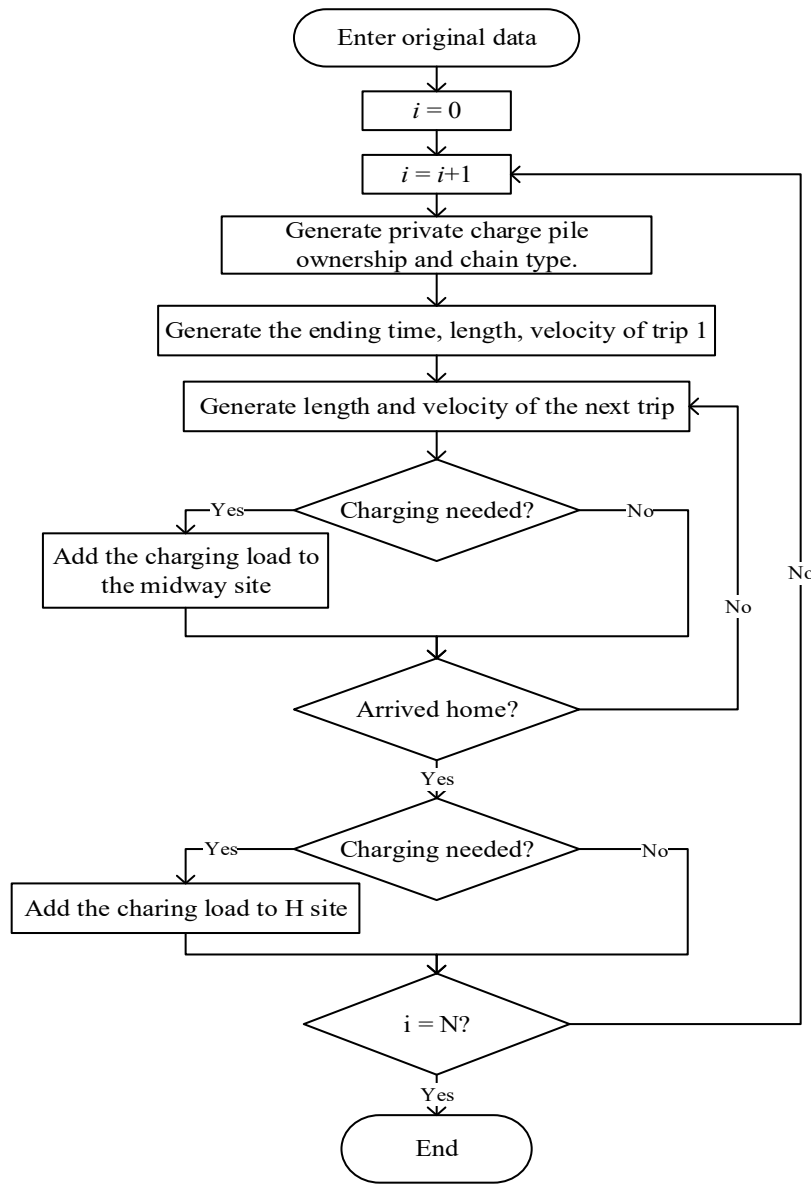

Fig. 2 Flow chart for Monte Carlo simulation procedure

After obtaining the day-ahead charging load curve of the five types of locations, the day-ahead charging load of a specific charging station can be obtained by

$$
p_{\text {station }}=\sum_{i=1}^{5} p_{i} \cdot r_{i}
$$

where $p_{i}$ is the load of the 5 sites, and $r_{i}$ is the function proportion of the 5 kinds of sites. For instance, $r_{1}=0.04$ means $4 \%$ home function of the whole studied area is accumulated in the service area of the station.

\section{Obtaining the actual maximum demand distribution}

Based on the Monte-Carlo-based charge load forecasting method, the charging load curve of the charging station within one day can be obtained. Since the charging power of EVs accounts for the main part of the power used by the charging station (especially for peak charging periods), the maximal value of the charging load in a day is regarded as the maximal demand of the charging station that day.

$\tilde{d}_{0}$ presents maximal daily demand predicted by Monte Carlo simulation. It meets the condition: $\tilde{d} \sim N\left(\tilde{d}_{0}, \sigma^{2}\right)$. Therefore, the probability density function of the maximal daily demand $g_{\tilde{d}}(\bullet)$ can be written as:

$$
g_{\tilde{d}}(d)=\frac{1}{\sigma \sqrt{2 \pi}} e^{-\frac{\left(d-\tilde{d}_{0}\right)^{2}}{2 \sigma^{2}}}
$$

However, the demand charge is charged according to monthly maximal demand Therefore, the distribution of monthly maximal demand needs to be derived based on the distribution of daily maximal demand. It is assumed that the daily maximal demand of each day in this month is an independent event and obeys the same distribution (10). Since the monthly maximal demand is also the maximal among the daily maximal demands, when $\Delta d$ approaches zero, the probability density function of monthly maximal demand $f_{\tilde{D}}(\bullet)$ satisfies:

$$
f_{\tilde{D}}(d) \Delta d=\left[G_{\tilde{d}}(d)\right]^{N}-\left[G_{\tilde{d}}(d-\Delta d)\right]^{N}
$$

where $G_{\tilde{d}}(\bullet)$ presents the cumulative distribution function of the daily maximal demand.

Equation (11) can be rewritten as:

$$
f_{\tilde{D}}(d)=\lim _{\Delta d \rightarrow 0} \frac{\left[G_{\tilde{d}}(d)\right]^{N}-\left[G_{\tilde{d}}(d-\Delta d)\right]^{N}}{\Delta d}
$$

It is observed that the right part of equation (12) conforms to the definition of derivative. Therefore, it can be expressed as:

$$
\begin{aligned}
f_{\tilde{D}}(d) & =\left[G_{\tilde{d}}(d)^{N}\right]^{\prime} \\
& =N \cdot G_{\tilde{d}}(d)^{N-1} \cdot G_{\tilde{d}}{ }^{\prime}(d) \\
& =N \cdot G_{\tilde{d}}(d)^{N-1} \cdot g_{\tilde{d}}(d)
\end{aligned}
$$

Equation (13) shows that the probability density function of monthly maximal demand can be obtained through the distribution of daily maximal demand.

\section{Case study}

\subsection{Load forecasting results}

In this case study, the meanings and values of input parameters are shown in Table 1. To obtain the charging load adapted to the actual situation, these input parameters can be adjusted.

Table 1. The parameters of the numerical case

\begin{tabular}{cc}
\hline Parameter & Value \\
\hline$p_{\text {own }}$ & $50 \%$ \\
$n_{E V}$ & 10000 \\
$p_{\text {charing }}$ & $60 \mathrm{~kW}$ \\
$c_{E V}$ & $40 \mathrm{kWh}$ \\
$u$ & $0.2 \mathrm{kWh} / \mathrm{km}$ \\
$r$ & {$[0.040 .10 .20 .10 .1]$} \\
$C$ & $2500 \mathrm{~kW}$ \\
\hline
\end{tabular}

The day-ahead charging load curve of the charging station based on the Monte Carlo simulation method is shown in Figure 3. 


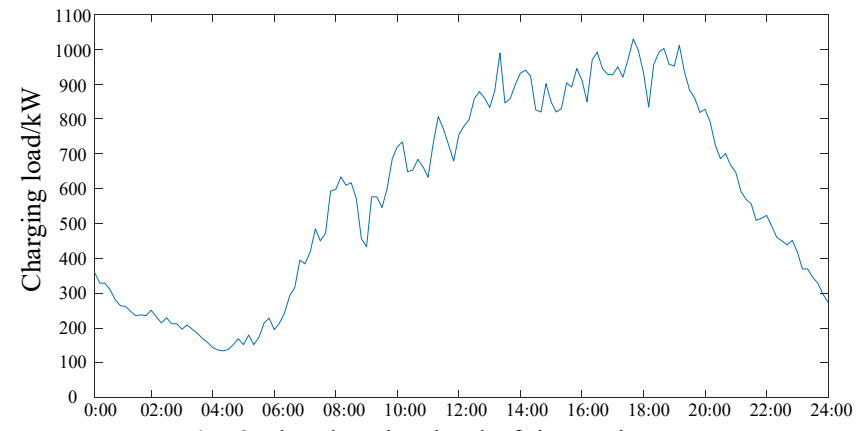

Fig. 3 The charging load of the station

Fig. 3 shows that the maximum of the charging load curve is 1020 , which is the predicted daily maximal demand.

\subsection{Contracting strategy}

The predicted daily maximal demand has been obtained in the section 4.1. Assuming that the standard deviation of the forecast error meets the condition: $\sigma=0.1 \tilde{d}_{0}$, the probability density function of the daily maximal demand can be expressed as:

$$
g_{\tilde{d}}(d)=0.003911 e^{-\frac{(d-1020)^{2}}{20808}}
$$

When the value of $N$ is 30 , the probability density function of the monthly maximal demand can be calculated through equation (13). Since the distribution of monthly maximal demand is complicated, it is not specifically listed here.

According to the demand contracting strategy described in (5), the optimal value of the contract demand can be obtained. Using the numerical calculation tool in MATLAB, it is calculated that:

$$
F_{\tilde{D}}^{-1}(0.524)=1226.793 \mathrm{~kW}
$$

which meets the condition: $F_{\tilde{D}}^{-1}(0.524)>0.42 C$. Hence, the optimal contract demand in this case study is $F_{\tilde{D}}^{-1}(0.524) / 1.05=1168.374 \mathrm{~kW}$. That means when the contract demand is set to $1168.374 \mathrm{~kW}$, the expectation of the demand charge will reach the minimum.

It should be pointed out that if the calculation result of equation (15) is less than $0.4 C=1000 \mathrm{~kW}$, the optimal value of the contract demand will be changed into $\underline{D}=0.4 C=1000 \mathrm{~kW}$.

\subsection{Comparison of two rules}

Besides charging according to the maximal demand, another way of calculating the basic electricity charge is according to the transformer capacity. Generally speaking, the unit price charged according to the transformer capacity is $2 / 3$ times that charged according to the maximal demand. For example, the basic electricity charge is $28 ¥ / \mathrm{kW}$ based on the transformer capacity, and $42 ¥ / \mathrm{kW}$ based on the maximal demand in Shanghai ${ }^{[17]}$.
For the charging stations in this case study, the basic electricity fee under different methods are compared, which are:

Method I: According to the transformer capacity.

Method II: According to the maximal demand, and setting the contract demand to minimal limit.

Method III: According to the maximal demand, and the contract demand following the demand contracting strategy proposed in this paper.

The results are shown in Table 2.

Table 2. The basic charge under three methods

\begin{tabular}{ccc}
\hline Method I/¥ & Method II/¥ & Method III/¥ \\
\hline 70000 & 51943.416 & 50809.105 \\
\hline
\end{tabular}

It can be seen that in this case study, the demand charge has a clear advantage in reducing the cost of electricity bills compared to the transformer capacity method. In addition, following the proposed demand contracting strategy can reduce the expectation of the demand charge effectively through this case study.

\section{Conclusion}

In this paper, a stochastic optimization model is established by regarding the actual maximal demand as a stochastic parameter, the object of which is to minimize the expectation of demand charge, and the analytic solution of the contract demand is derived.

For the distribution of the actual maximal demand, this paper proposes a Monte Carlo simulation method based on the trip chain theory to predict the charging load of the charging station. This method can obtain the distribution of the daily maximal demand combined with the actual situation of charging stations. In addition, this paper proposes the method of obtaining the monthly maximal demand based on the daily maximal demand to complete the demand contracting strategy for charging stations. It uses a virtual charging station as the case study to show the whole process of the demand contracting strategy for charging stations. The results of the case study illustrate the feasibility of this strategy.

The most innovative point of this article is that in face of the basic demand charge added to the electricity cost of charging stations in the future, it proposes the demand contracting strategy for charging stations correspondingly. Compared with the existing research, this strategy is involved in the demand charge that is rarely discussed. It has the guiding significance for the operators of charging stations to predict the charging load and revise the contract demand in time.

The direction of the future research is to explore the relationship between the charging load of charging stations with a certain operating history and many factors such as time, weather, holidays, special events, etc. In this way, a more accurate forecasting distribution of the maximal demand can be acquired, and the demand contracting strategy can be further improved to reduce the electricity cost. 


\section{Acknowledgement}

The authors gratefully acknowledge the support from the Technology Program of SGCC under Grant 52094018002P.

\section{References}

1. Ministry of Industry and Information Technology of the People's Republic of China. Public consultation on the "New Energy Automobile Industry Development Plan (2021-2035)" [EB/OL]. (201912-03)[2020-03-16] (in Chinese). Available:

http://www.miit.gov.cn/n1278117/n1648113/c75536 23/content.html

2. BEHEV (2007), Introduction of Electric Vehicle Policies in Other Countries (in Chinese) . Available: http://www.bjhev.com/ReadNews.asp?NewsID=122 0

3. H. Chen, J. Jin and J. Chen, "Catching up in new energy vehicle industry: Review of its development and policies in China," 2008 4th IEEE International Conference on Management of Innovation and Technology, Bangkok, 2008, 810-814.

4. Virginia Electric and Power Company. Schedule 6 Large General Service. [Online]. Available: https://www.dom.com/

5. Uttar Gujarat VIJ Company Limited. Tariff for Supply of Electricity at Low Tension, High Tension and Extra High Tension. [Online]. Available: http://www.ugvcl.com

6. The National Development and Reform Commission of the People's Republic of China. Notice on Issues Concerning Electric Vehicle Electricity Price Policy. [EB/OL]. (2014-08-06) [2020-03-16] (in Chinese). Available:

https://www.ndrc.gov.cn/fggz/tzgg/ggkx/201408/t20 140806_1073810.html

7. The National Development and Reform Commission of the People's Republic of China. Notice of the National Development and Reform Commission on the Implementation Measures for the Reform of Printing Electricity Price.[EB/OL]. (2005-03-28) [2020-03-16] (in Chinese). Available: https://www.ndrc.gov.cn/xxgk/zcfb/tz/200506/t2005 0613_965813.html

8. H. Zhang. Strengthen DSM to implement maximum power load demand control[J]. Power Demand Side Management, 2005, 7(3):55-56, 63 (in Chinese).

9. X. Chen. Problems and solutions to bi-lateral electricity price in sales side[J]. Power Demand Side Management, 2009, 11(5):57-60 (in Chinese).

10. E. Sortomme and M. A. El-Sharkawi . Optimal charging strategies for unidirectional vehicle-togrid[J]. IEEE Trans. on Smart Grid, 2011, 2(1): 131-138.
11. K. Clement-Nyns, E. Haesen and J. Driesen. The impact of charging plug in hybrid electric vehicles on a residential distribution grid[J]. IEEE Trans. on Power Systems, 2010, 25(1): 371-380.

12. T. H. Bradley, C. W. Quinn. Analysis of plug-in hybrid electric vehicle utility factors[J]. Journal of Power Sources, 2010, 195 (16): 5399-5408.

13. P. H. Andersen, M. Rask, J. A. Mathews. Integrating private transport into renewable energy policy: the strategy of creating intelligent recharging grids for electric vehicles[J]. Energy Policy, 2009, 37(7): 2481-2486.

14. S. W. Hadley, A. Tsvetkova. Potential impacts of plug-in hybrid electric vehicles on regional power generation[R]. Tennessee: Oak Rige National Laboratory, 2008.

15. O. Marcincin, Z. Medvec and P. Moldrik, "The impact of electric vehicles on distribution network," 2017 18th International Scientific Conference on Electric Power Engineering (EPE), Kouty nad Desnou, 2017 : 1-5.

16. Z. Liu, D. Feng, F. Wu, Y.Zhou, C. Fang. Contract Demand Decision for Electricity Users with Stochastic Photovoltaic Generation. Proceeding of the CSEE, 2020, 40(6): 1865-1872(in Chinese).

17. State Grid Shanghai Electric Power Company. Shanghai Electricity Price List [EB/OL]. (2019-0531) [2020-03-16] (in Chinese). Available: http://www.95598.cn/static/html//person/sas/es//PM 06003001_2352.shtml 